\title{
T4-like phages reveal the potential role of viruses in soil organic matter mineralization
}

Xiaomeng Wei ${ }^{a}$, Tida $G e^{a, b^{*}}$, Chuanfa $\mathrm{Wu}^{b}$, Shuang Wang ${ }^{b}$, Kyle Mason-Jones ${ }^{c}$, Yong $\mathrm{Li}^{d}$, Zhenke Zhu ${ }^{a}$, Yajun $\mathrm{Hu}^{a}$, Chao Liang ${ }^{e}$, JianLin Shen ${ }^{a}$, Jinshui $^{\mathrm{W}}{ }^{a}{ }^{a}$, Yakov Kuzyakov ${ }^{f, g}$

${ }^{a}$ Key Laboratory of Agro-ecological Processes in Subtropical Region \& Changsha Research Station for Agricultural and Environmental Monitoring, Institute of Subtropical Agriculture, Chinese Academy of Sciences, Hunan 410125, PR China

${ }^{\mathrm{b}}$ State Key Laboratory for Managing Biotic and Chemical Threats to the Quality and Safety of Agro-products, Key Laboratory of Biotechnology in Plant Protection of Ministry of Agriculture and Zhejiang Province, Institute of Plant Virology, Ningbo University, Ningbo 315211, PR China

${ }^{\mathrm{c}}$ Department of Terrestrial Ecology, Netherlands Institute of Ecology (NIOOKNAW), Wageningen, 106708, The Netherlands

${ }^{\mathrm{d}}$ Zhejiang Provincial Key Laboratory of Agricultural Resources and Environment, College of Environmental and Resource Sciences, Zhejiang University, Hangzhou 310058, PR China

${ }^{\mathrm{e}}$ Institute of Applied Ecology, Chinese Academy of Sciences, Shenyang, 110016, PR China 
${ }^{\mathrm{f}}$ Department of Soil Science of Temperate Ecosystems, Department of Agricultural

Soil Science, University of Goettingen, Goettingen, 37073, Germany

g Agro-Technological Institute, RUDN University, 117198 Moscow, Russia 
Table S1 SOC mineralization rate (shown as $\mathrm{CO}_{2}$ efflux) and DOC content. NoS: nonsterilized soil; NoI: non-inoculated sterilized soil; LowI: low-inoculated sterilized soil; HighI: high-inoculated sterilized soil. $P$ values of the pairwise comparison between treatments at each sampling time are shown in Table S3.

\begin{tabular}{llllll}
\hline & & 1 week & 2 weeks & 4 weeks & 6 weeks \\
\hline \multirow{4}{*}{$\mathrm{CO}_{2}$ efflux } & NoS & 28.7 & 19.6 & 25.2 & 7.5 \\
& NoI & 37.4 & 22.4 & 22.3 & 11.4 \\
& LowI & 72.8 & 30.0 & 15.9 & 16.2 \\
& HighI & 83.5 & 38.8 & 12.8 & 18.1 \\
\multirow{4}{*}{ DOC } & NoS & 106.0 & 100.4 & 112.7 & 129.1 \\
& NoI & 442.8 & 435.6 & 450.1 & 436.2 \\
& LowI & 391.3 & 396.8 & 434.6 & 408.8 \\
& HighI & 351.3 & 352.2 & 370.9 & 353.4 \\
\hline
\end{tabular}


Table S2 Total 16S rRNA and $g 23$ abundance and the abundance of 16S rRNA and $g 23$ after PMA applied. NoS: non-sterilized soil; NoI: non-inoculated sterilized soil; LowI: low-inoculated sterilized soil; HighI: high-inoculated sterilized soil. $P$ values of the pairwise comparison between treatments at each sampling time are shown in Table S3.

\begin{tabular}{|c|c|c|c|c|c|c|}
\hline & & 0 week & 1 week & 2 week & 4 week & 6 week \\
\hline \multirow{5}{*}{$\begin{array}{c}\text { Total } \\
\text { 16S rRNA }\end{array}$} & NoS & $1.1 \mathrm{e} \times 10^{10}$ & $1.4 \times 10^{10}$ & $1.0 \times 10^{10}$ & $9.1 \times 10^{9}$ & $3.0 \times 10^{9}$ \\
\hline & NoI & $2.0 \mathrm{e} \times 10^{9}$ & $2.3 \times 10^{9}$ & $1.1 \times 10^{9}$ & $6.9 \times 10^{9}$ & $2.2 \times 10^{9}$ \\
\hline & LowI & $2.2 \mathrm{e} \times 10^{9}$ & $4.3 \times 10^{9}$ & $7.2 \times 10^{9}$ & $2.9 \times 10^{9}$ & $2.4 \times 10^{9}$ \\
\hline & HighI & $2.3 \mathrm{e} \times 10^{9}$ & $1.4 \times 10^{10}$ & $1.1 \times 10^{10}$ & $1.9 \times 10^{9}$ & $8.8 \times 10^{9}$ \\
\hline & NoS & $8.3 \times 10^{9}$ & $1.0 \times 10^{10}$ & $6.2 \times 10^{9}$ & $2.5 \times 10^{9}$ & $1.1 \times 10^{9}$ \\
\hline \multicolumn{2}{|c|}{ PMA-applied NoI } & $1.5 \times 10^{9}$ & $1.9 \times 10^{9}$ & $9.2 \times 10^{8}$ & $5.7 \times 10^{8}$ & $1.2 \times 10^{9}$ \\
\hline \multirow[t]{2}{*}{ 16S rRNA } & LowI & $2.3 \times 10^{9}$ & $1.9 \times 10^{9}$ & $2.7 \times 10^{9}$ & $9.2 \times 10^{8}$ & $1.4 \times 10^{9}$ \\
\hline & HighI & $2.2 \times 10^{9}$ & $1.0 \times 10^{10}$ & $1.8 \times 10^{9}$ & $1.8 \times 10^{9}$ & $3.5 \times 10^{9}$ \\
\hline \multirow{4}{*}{ Total g23 } & NoS & $1.4 \times 10^{6}$ & $7.0 \times 10^{5}$ & $6.7 \times 10^{6}$ & $1.7 \times 10^{7}$ & $1.0 \times 10^{7}$ \\
\hline & $\mathrm{NoI}$ & $1.2 \times 10^{4}$ & $1.2 \times 10^{5}$ & $1.2 \times 10^{7}$ & $1.2 \times 10^{7}$ & $1.2 \times 10^{7}$ \\
\hline & LowI & $2.8 \times 10^{4}$ & $1.8 \times 10^{4}$ & $1.2 \times 10^{7}$ & $2.6 \times 10^{7}$ & $2.6 \times 10^{7}$ \\
\hline & HighI & $3.5 \times 10^{4}$ & $2.5 \times 10^{4}$ & $1.2 \times 10^{7}$ & $1.1 \times 10^{7}$ & $1.3 \times 10^{7}$ \\
\hline & NoS & $8.6 \times 10^{5}$ & $4.6 \times 10^{5}$ & $4.7 \times 10^{6}$ & $1.4 \times 10^{7}$ & $7.6 \times 10^{6}$ \\
\hline \multicolumn{2}{|c|}{ PMA-applied NoI } & $9.3 \times 10^{3}$ & $2.5 \times 10^{4}$ & $6.9 \times 10^{6}$ & $1.2 \times 10^{7}$ & $3.2 \times 10^{6}$ \\
\hline \multirow[t]{2}{*}{ g23 } & LowI & $1.8 \times 10^{4}$ & $2.1 \times 10^{4}$ & $7.6 \times 10^{6}$ & $2.3 \times 10^{7}$ & $7.3 \times 10^{6}$ \\
\hline & HighI & $2.8 \times 10^{4}$ & $1.3 \times 10^{4}$ & $8.9 \times 10^{6}$ & $1.1 \times 10^{7}$ & $1.1 \times 10^{7}$ \\
\hline
\end{tabular}


Table S3 $P$ values of the pairwise comparison between treatments at each sampling time of SOC mineralization rate (shown as $\mathrm{CO}_{2}$ efflux) and DOC content, and total $16 \mathrm{~S}$ rRNA and $g 23$ abundance and the abundance of 16S rRNA and $g 23$ after PMA applied. NoS: non-sterilized soil; NoI: non-inoculated sterilized soil; LowI: low-inoculated sterilized soil; HighI: high-inoculated sterilized soil

\begin{tabular}{|c|c|c|c|c|c|c|}
\hline & HighI-LowI & HighI-NoI & HighI-NoS & LowI-NoI & LowI-NoS & NoI-NoS \\
\hline DOC-week1 & 0.067 & 0.001 & $<0.001$ & 0.023 & $<0.001$ & $<0.001$ \\
\hline DOC-week2 & 0.011 & $<0.001$ & $<0.001$ & 0.022 & $<0.001$ & $<0.001$ \\
\hline DOC-week4 & 0.002 & $<0.001$ & $<0.001$ & 0.349 & $<0.001$ & $<0.001$ \\
\hline DOC-week6 & $<0.001$ & $<0.001$ & $<0.001$ & 0.010 & $<0.001$ & $<0.001$ \\
\hline $\mathrm{CO}_{2}$ efflux-week1 & 0.001 & $<0.001$ & $<0.001$ & 0.000 & 0.000 & 0.003 \\
\hline $\mathrm{CO}_{2}$ efflux-week2 & $<0.001$ & $<0.001$ & $<0.001$ & 0.001 & 0.000 & 0.128 \\
\hline $\mathrm{CO}_{2}$ efflux-week4 & 0.129 & $<0.001$ & $<0.001$ & 0.003 & $<0.001$ & 0.105 \\
\hline $\mathrm{CO}_{2}$ efflux-week6 & 0.056 & $<0.001$ & $<0.001$ & $<0.001$ & $<0.001$ & $<0.001$ \\
\hline $\begin{array}{l}\text { Total 16S rRNA- } \\
\text { week0 }\end{array}$ & 0.036 & $<0.001$ & $<0.001$ & 0.002 & $<0.001$ & $<0.001$ \\
\hline $\begin{array}{l}\text { Total 16S rRNA- } \\
\text { week1 }\end{array}$ & $<0.001$ & $<0.001$ & 0.880 & 0.867 & $<0.001$ & $<0.001$ \\
\hline $\begin{array}{l}\text { Total 16S rRNA- } \\
\text { week2 }\end{array}$ & 0.013 & $<0.001$ & 0.839 & $<0.001$ & 0.019 & $<0.001$ \\
\hline $\begin{array}{l}\text { Total } 16 \mathrm{~S} \text { rRNA- } \\
\text { week4 }\end{array}$ & $<0.001$ & $<0.001$ & 0.003 & 0.135 & 0.000 & $<0.001$ \\
\hline $\begin{array}{l}\text { Total } 16 \mathrm{~S} \text { rRNA- } \\
\text { week6 }\end{array}$ & $<0.001$ & $<0.001$ & $<0.001$ & 0.804 & 0.539 & 0.393 \\
\hline $\begin{array}{l}\text { PMA-applied 16S } \\
\text { rRNA-week0 }\end{array}$ & 0.493 & 0.003 & $<0.001$ & 0.002 & $<0.001$ & $<0.001$ \\
\hline $\begin{array}{l}\text { PMA-applied 16S } \\
\text { rRNA-week1 }\end{array}$ & $<0.001$ & $<0.001$ & 0.899 & 0.352 & $<0.001$ & $<0.001$ \\
\hline $\begin{array}{l}\text { PMA-applied 16S } \\
\text { rRNA-week2 }\end{array}$ & 0.078 & 0.082 & $<0.001$ & 0.002 & $<0.001$ & $<0.001$ \\
\hline $\begin{array}{l}\text { PMA-applied 16S } \\
\text { rRNA-week4 }\end{array}$ & 0.002 & 0.000 & 0.012 & 0.154 & $<0.001$ & $<0.001$ \\
\hline $\begin{array}{l}\text { PMA-applied 16S } \\
\text { rRNA-week6 }\end{array}$ & $<0.001$ & $<0.001$ & $<0.001$ & 0.550 & 0.252 & 0.567 \\
\hline g23-week0 & 0.000 & 0.525 & 0.115 & $<0.001$ & $<0.001$ & 0.318 \\
\hline g23-week1 & 0.087 & 0.160 & 0.669 & 0.006 & 0.040 & 0.310 \\
\hline g23-week2 & 0.007 & 0.000 & 0.000 & 0.000 & 0.002 & 0.011 \\
\hline g23-week4 & 0.225 & 0.580 & 1.000 & 0.491 & 0.225 & 0.580 \\
\hline g23-week6 & 0.003 & 0.018 & 0.452 & 0.386 & 0.001 & 0.004 \\
\hline $\begin{array}{l}\text { PMA-applied g23- } \\
\text { week0 }\end{array}$ & 0.493 & 0.003 & 0.000 & 0.002 & $<0.001$ & $<0.001$ \\
\hline $\begin{array}{l}\text { PMA-applied g23- } \\
\text { week1 }\end{array}$ & $<0.001$ & $<0.001$ & 0.899 & 0.352 & $<0.001$ & $<0.001$ \\
\hline $\begin{array}{l}\text { PMA-applied } g 23- \\
\text { week2 }\end{array}$ & 0.078 & 0.082 & $<0.001$ & 0.002 & $<0.001$ & $<0.001$ \\
\hline
\end{tabular}


PMA-applied g23week 4

0.002

$<0.001$

0.012

0.154

$<0.001$

$<0.001$

PMA-applied g23-

$<0.001$

$<0.001$

$<0.001$

0.550

0.252

0.567 
Fig. S1 Gel picture of DNA extracted from soils incubated for 0,1 and 2 weeks after $\gamma$ radiation sterilization. The gel was prepared used $1 \%$ agarose and run at $100 \mathrm{~V}$ for 30 min using $\lambda$ DNA-HindIII as the marker, the first band of which is as long as $23130 \mathrm{bp}$ and provide an indicator of little damaged genomic DNA fragments. The DNA concentration immediately after the sterilization $\left(0,4.5 \pm 0.3 \mathrm{ug} \mathrm{g}^{-1}\right.$, nrep=3) was similar to that in the non-sterilized soil $\left(4.3 \pm 0.4 \mathrm{ug} \mathrm{g}^{-1}\right.$, nrep=3), and decreased to near 0 after 2-week incubation $\left(0.6 \pm 0.1 \mathrm{ug} \mathrm{g}^{-1}\right.$, nrep=3).

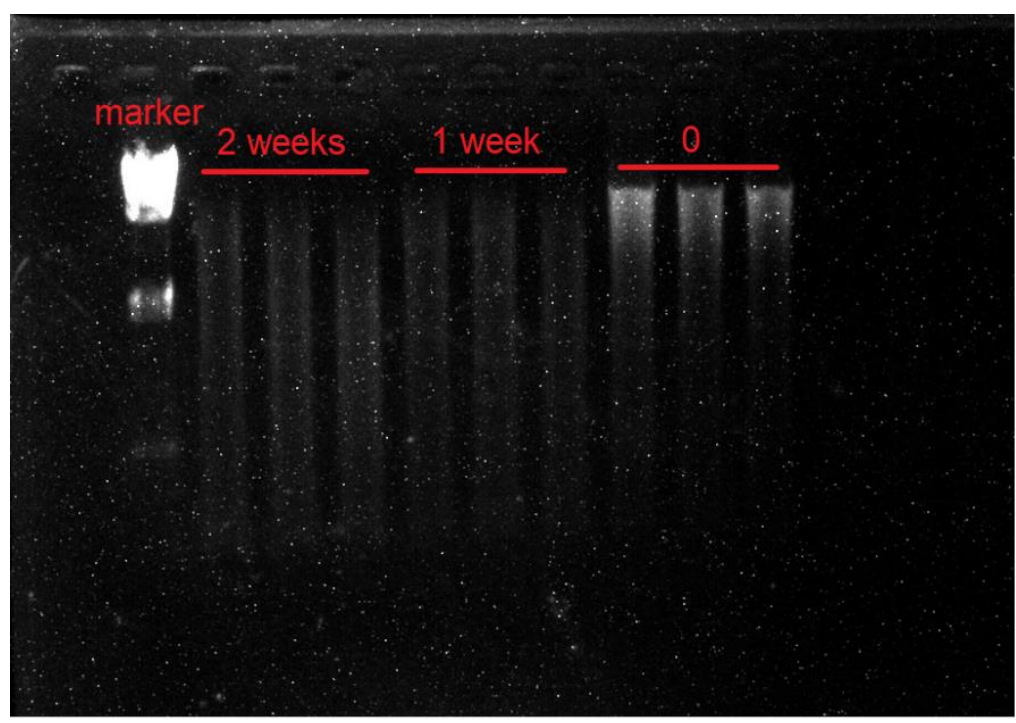


Fig. S2 Pearson correlation between DOC, C mineralization rate (C Min rate), total 16S rRNA and $g 23$ abundance and the abundance of 16S rRNA and $g 23$ after PMA applied.

\begin{tabular}{|c|c|c|c|c|c|}
\hline DOC & & 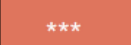 & $\star \star \star *$ & & \\
\hline 0.2 & C Min rate & * & $\star * *$ & $\star * *$ & $* * *$ \\
\hline-0.54 & 0.31 & $\begin{array}{c}\text { Total } \\
\text { 16S rRNA }\end{array}$ & *** & * & \\
\hline-0.48 & 0.42 & 0.84 & $\begin{array}{c}\text { PMA-applied } \\
16 S \text { rRNA }\end{array}$ & $* * *$ & $* * *$ \\
\hline 0.18 & -0.58 & $=0.32$ & -0.51 & Total g23 & *** \\
\hline & -0.48 & & -0.43 & 0.77 & $\begin{array}{c}\text { PMA-applied } \\
\text { g23 }\end{array}$ \\
\hline
\end{tabular}


Fig. S3 Linear regressions of the total 16S rRNA gene abundance (a, d), PMA-applied $16 \mathrm{~S}$ rRNA gene abundance $(\mathrm{b}, \mathrm{e})$ and $16 \mathrm{~S}$ rRNA gene viable ratio $(\mathrm{c}, \mathrm{f})$ to $g 23$ abundance without (total, in orange color) and with PMA application (PMA-applied, in green color) in non-sterilized (a-c) and reinoculated sterilized soil (e-f). Trend lines are plotted using all treatments, with separate lines for PMA-applied and non-applied samples. Treatments in the plot are indicated by shapes of point, while significant levels of the regression are indicated by line types: Dotted line: $p<0.05$; solid line: $p<0.01$. NoS: non-sterilized soil; NoI: non-inoculated sterilized soil; LowI: low-inoculated sterilized soil; HighI: high-inoculated sterilized soil.
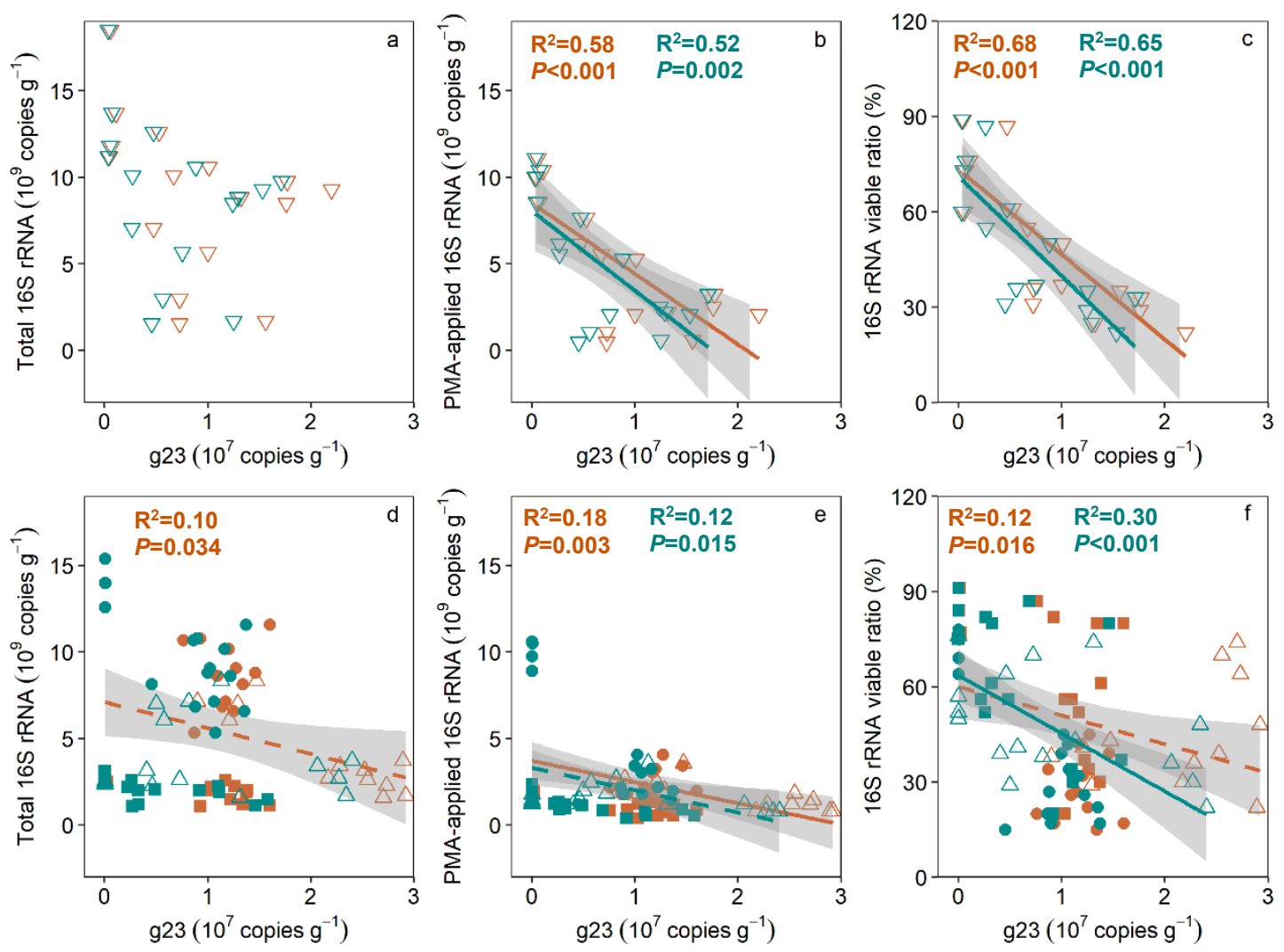

$\nabla$ NoS $\square$ Nol $\triangle$ Lowl $\bullet$ Highl $($ Total)
$\nabla$ NoS $\square$ Nol $\triangle$ Lowl $\bullet$ Highl $($ PMA-applied) 